\title{
Robotic surgical system under scrutiny
}

A fter more than a decade of spectacular growth and an expanding presence in US operating rooms, the world's most popular robotic surgical system has come under scrutiny.

Intuitive Surgical, Inc., maker of the da Vinci Surgical System, is facing questions about their marketing practices and lawsuits alleging injuries. The company has also received attention from the US Food and Drug Administration (FDA) about reports of alleged adverse events. As of Nov. 3, the FDA had received 3697 reports this year involving the da Vinci system, up from 1595 in 2012.

"A recent rise in MDRs [medical device reports] does not necessarily represent a recent spike in adverse events - all it means is that Intuitive Surgical was recently informed of a number of adverse events that could have occurred at any time in the past," says Lauren Burch, an Intuitive corporate communications representative.

The FDA agrees that the increase is partly the result of more physicians reporting. In July, however, it warned the company to correct alleged violations or face equipment seizures or fines after a report found the company had not adequately reported adverse events and product corrections. On Nov. 8, the FDA released the results of a survey of a small sample of surgeons about the safety and performance of the da Vinci system. Eleven surgeons responded and indicated that they thought robotic surgery benefitted patients in many ways, including reductions in bleeding, complications, recovery times, pain and lengths of hospital stay. But they also reported that "learning how to use the da Vinci Surgical System is the biggest challenge because of the device's complex user-interface."

Physicians and hospitals are required to report suspected medical devicerelated injuries or deaths to the FDA and the manufacturer. Manufacturers are required to report to the FDA when a malfunctioning device poses a threat or when a device may have caused or contributed to a death or injury. But this self-reporting system is "haphazard and inconsistent" and the law enforcing it

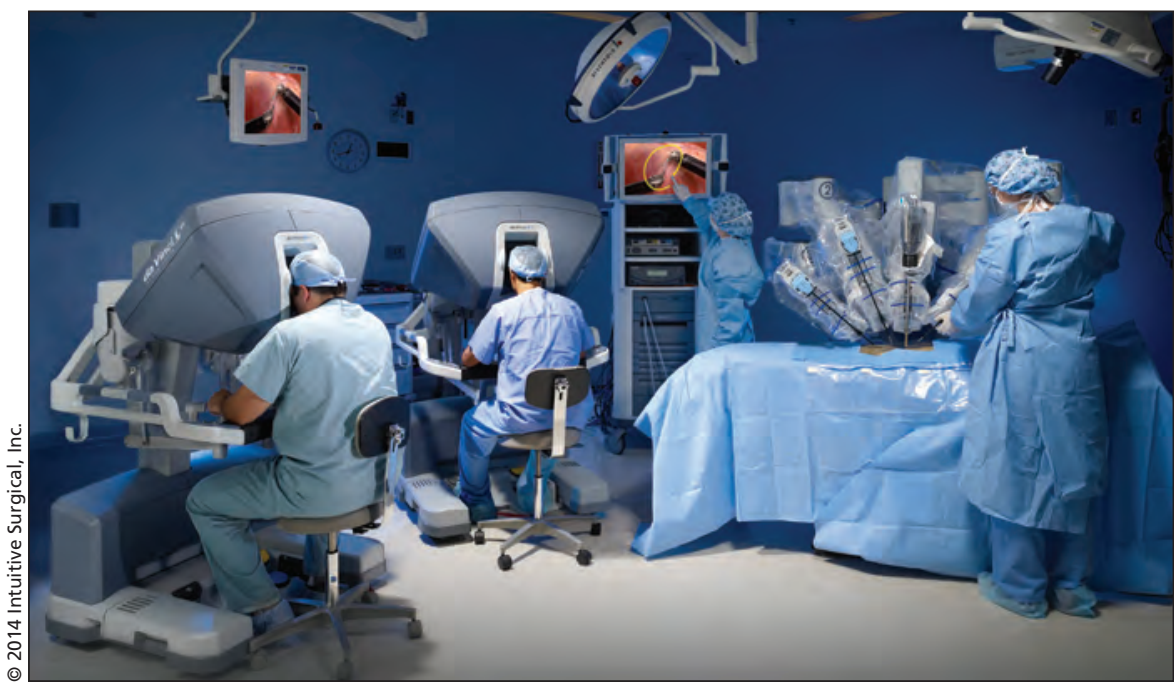

As of Nov. 3, the FDA had received 3697 reports of alleged adverse events this year involving the da Vinci Surgical System, up from 1595 in 2012.

has "no teeth," says Dr. Martin Makary, an associate professor of surgery at Johns Hopkins University School of Medicine in Baltimore, Maryland.

Makary contributed to a recent review of nearly 12 years of FDA data concerning adverse reports involving robotassisted laparoscopic surgery, finding 245 adverse incidents, including 71 deaths and 174 serious injuries (J Healthc Qual 2013 Aug 27. doi: 10.1111/jhq.12036).

"It's highly representative of a larger problem in health care," he says. "We lack standardized monitoring of new technology to properly evaluate it for safety and efficacy."

Robotic surgical technology has been "broadly adopted not based on good science but rather on patient demand and physician interests to attract patients," adds Makary. Getting accurate information to the public is essential as more Americans opt for the high-tech choice, Makary says. According to the study, the number of da Vinci systems in the US increased from 800 in 2007 to 1400 in 2011.

Hospitals and surgeons considering the purchase of a system should view the promotional material that accompanies it with a degree of skepticism, warns Makary. In an earlier analysis of US hospital websites, he found that a third claimed robotic surgery improved cancer outcomes - a finding "unsupported by medical literature," says Makary.
"None of the informational material had any mention of risks," he says. "People tend to view this as the doctor's voice, when, in many cases, we found the manufacturer authored much of the unsupported claims."

Makary also found that 73 of the websites used wording taken directly from Intuitive's resource material. Intuitive said in an email that it can't control how hospitals market the da Vinci device, and that the informational material it provides is "fair and balanced."

Another emerging criticism of robotic surgery is that it may not be the cost-effective solution hospitals were hoping it would be. For example, robotic surgery does not substantially reduce complication rates, transfusion requirements or rate of discharge to nursing facilities for hysterectomies, according to a recent study (JAMA 2013;309:68998). Although less likely to result in hospital stays longer than two days, the robotic procedures cost $\$ 2189$ more than traditional laparoscopic hysterectomies. - Patricia Guthrie, Seattle, WA.

CMAJ 2014. DOI:10.1503/cmaj.109-4648

\section{More News online}

To read more CMAJ news articles, visit cmaj.ca/site/home/news.xhtml 